\title{
Théologiques
}

Théologiques

\section{Une église au coeur de la ville}

\section{André Lamoureux}

Volume 3, numéro 1, mars 1995

Symbolique urbaine et foi chrétienne

URI : https://id.erudit.org/iderudit/602418ar

DOI : https://doi.org/10.7202/602418ar

Aller au sommaire du numéro

Éditeur(s)

Faculté de théologie de l'Université de Montréal

ISSN

1188-7109 (imprimé)

1492-1413 (numérique)

Découvrir la revue

\section{Citer cet article}

Lamoureux, A. (1995). Une église au coeur de la ville. Théologiques, 3(1),

115-127. https://doi.org/10.7202/602418ar

\section{Résumé de l'article}

Dans une société en mutation radicale et rapide, il est impérieux de repenser le rôle des grands édifices religieux en milieu urbain. Dans le texte qui va suivre, on ne trouvera pas une démarche théologique. J'ai simplement voulu répondre à un certain nombre de questions qu'on m'a posées à titre de responsable d’une grande église en milieu urbain. d'utilisation que vous pouvez consulter en ligne.

https://apropos.erudit.org/fr/usagers/politique-dutilisation/ 


\title{
Une église au cœur de la ville
}

\author{
André LAMOUREUX \\ curé de la paroisse Saint-Jean-Baptiste de Montréal
}

\section{RÉSUMÉ}

Dans une société en mutation radicale et rapide, il est impérieux de repenser le rôle des grands édifices religieux en milieu urbain. Dans le texte qui va suivre, on ne trouvera pas une démarche théologique. J'ai simplement voulu répondre à un certain nombre de questions qu'on m'a posées à titre de responsable d'une grande église en milieu urbain.

Au moment où j'allais accepter de prendre charge de la paroisse Saint-Jean-Baptiste, j'ai passé plusieurs heures à me promener dans ce quartier qui va du Mont-Royal au Parc Lafontaine. Je me suis arrêté devant l'église monumentale qui se dresse dans ce milieu populaire au cœur de la ville. Les interrogations et les réflexions se sont précipitées dans mon esprit.

D'abord, j'étais impressionné par l'immensité de ce temple qui confinait presque à la démesure. Devant la splendeur de l'architecture et tant de beautés, je trouvais émouvant et redoutable de me voir confier, en solidarité avec les fidèles, un tel patrimoine, un si bel héritage de foi et de culture.

Quels défis aurions-nous à relever? De quelles ressources disposerions-nous? Il était difficile de se faire une idée des problèmes et des possibilités qui allaient déterminer nos responsabilités à venir.

Une fois en contact quotidien avec la réalité du milieu, en lien avec ceux et celles qui la vivent, on ne peut s'empêcher de penser que la pertinence du maintien d'un tel temple est loin d'être évidente pour tous. Lorsque l'église actuelle, la troisième depuis la fondation de la paroisse, a été inaugurée en 1915, en fonction de rassemblements qui réunissaient plusieurs fois par semaine près de trois mille fidèles, ses dimensions 
correspondaient aux besoins du temps et à la vitalité d'une communauté qui ne faisait qu'un avec le quartier.

Les temps ont changé. La fréquentation de l'église et la pratique sacramentelle ont connu une baisse plus que considérable. Parallèlement à ce phénomène, le quartier, hier stable et habité par des gens qui se connaissaient entre eux, est désormais marqué par des réalités nouvelles, tout à fait inédites. Nous sommes en pleine mouvance. La population se renouvelle pratiquement tous les cinq ans. Les anciens vivent ce qu'on pourrait appeler un certain exil intérieur. Ils peuvent demeurer dans la maison de leur enfance tout en étant entourés d'étrangers. D'un côté, ils se sentent chez eux, avec des points de repère familiers. De l'autre, ils vivent en terre étrangère, en contact avec des citoyens qui ne viennent pas s'installer à vie. Le caractère multi-ethnique de cette partie du Plateau Mont-Royal ajoute au dépaysement. Là où hier se trouvaient presque exclusivement des Québécois de langue française, aujourd'hui se côtoient des gens venus de tous les coins du monde. L'école élémentaire et le collège privé du milieu n'ont rien de commun avec les souvenirs d'enfance. Cette situation de multi-ethnicité a marqué un bon nombre de quartiers de la ville.

\section{Quel sens peut prendre un grand lieu de culte dans un tel contexte?}

Il ne manque pas de théoriciens et d'observateurs qui nous proposent des solutions du genre "prêt à porter ", solutions souvent courtes auxquelles ils donnent des airs d'évidence : il conviendrait de vendre nos édifices, il faudrait les démolir, etc. Quand on veut se livrer à une réflexion sérieuse vraiment respectueuse de la réalité, il faut bâtir à partir du milieu un réseau de gens expérimentés et capables de vision. C'est pourquoi j'ai accepté de partager ici un peu de ce que j'ai appris sur le terrain, comme on le fait lorsqu'on assume une responsabilité complexe ${ }^{1}$.

Je veux tenir compte des problèmes qui provoquent les remises en cause nécessaires, mais aussi des attentes qui se sont exprimées et qui nous laissent entrevoir de nouveaux usages des espaces paroissiaux et l'émergence d'un nouveau type de rapport entre la communauté chrétienne, son église, ses locaux, et la société. On verra s'il faut réinventer le rôle des

1 Je remercie Guy Bérubé, mon vicaire qui partage ma tâche pastorale. Nos questionnements et nos échanges ont inspiré ces réflexions. Je dois beaucoup également à Alain Faubert qui a bien voulu m'aider à relire mon expérience et à préciser ma pensée. 
grands édifices religieux en milieu urbain et en conséquence passer à l'action avec ce que cela suppose de créativité.

Peut-on parler d'une nouvelle signification d'une grande église patrimoniale dans la cité contemporaine? Pour répondre à cette question décisive pour l'avenir de l'Église en contexte urbain, je livrerai mes réflexions en trois temps, en m'appuyant sur mon expérience de pasteur placé quotidiennement devant le défi de faire servir et rayonner un haut lieu auprès de toutes les personnes et de tous les groupes qui peuvent le fréquenter.

Nous allons d'abord explorer les attentes, les demandes et les besoins: la réalité vécue autour d'un lieu religieux en effervescence comme Saint-Jean-Baptiste de Montréal peut être éclairante. Nous nous attarderons ensuite à décrire les réactions, les ouvertures et les convictions de l'équipe pastorale en place devant la diversification des usages d'un espace religieux traditionnellement réservé aux événements du culte. Enfin, une troisième partie de cet article présentera quelques pistes ecclésiologiques et pastorales susceptibles d'alimenter une réflexion plus approfondie sur le sujet.

\section{Des attentes diversifiées}

Il est peut-être intéressant de souligner quelques attentes qui nous sont exprimées quant à l'utilisation des lieux. Malgré bien des ennuis - comme par exemple le manque de personnel et les difficultés de stationnement -, les espaces actuels attirent les gens et rendent possibles des initiatives et des événements de divers ordres.

À mon arrivée il y a trois ans, quelques institutions occupaient déjà une partie du sous-sol. J'ai rencontré des personnes compétentes et profondément engagées socialement, des services de première ligne à base de bénévolat et de participation du plus grand nombre. Le Centre $\mathrm{Au}$ Puits, avec son équipe de travailleuses sociales, s'avère quasi indispensable pour une centaine de personnes seules, démunies, qui apprennent l'estime d'elles-mêmes et l'autonomie à travers leurs contacts et leurs activités. La Saint-Vincent-de-Paul n'a pas, bien sûr, la prétention de remplacer les services sociaux. Cependant, elle accueille dans ses locaux un grand nombre de personnes en détresse, dans le respect de leur dignité et en tenant compte de l'urgence de leurs besoins immédiats bien concrets. Enfin, la Mission de langue espagnole est fortement établie chez nous depuis une vingtaine d'années. Elle utilise toujours de vastes locaux de même que l'église et la chapelle pour l'ensemble de ses célébrations. En plus de rassembler chaque semaine des centaines d'espagnols et de latino-américains, elle accueille un grand nombre d'immigrants et assure 
une distribution de nourriture à cent cinquante familles chaque semaine. Ses responsables offrent une grande variété de cours et d'activités communautaires. La présence de cette communauté apporte un dynamisme particulier qui contribue à faire de notre temple un lieu vivant de rassemblement, d'évangélisation et de culture.

Aujourd'hui, la liste des services s'allonge au rythme des demandes: cuisines collectives, popote roulante, loisirs pour les jeunes, etc. Beaucoup de groupes ou de familles réservent des salles pour une réception ou une grande rencontre. Le CLSC, par exemple, y organise son grand souper de Noël pour les personnes âgées. Les groupes communautaires, les services publics et parapublics, de même que les réseaux de concertation et de collaboration du quartier sont familiers avec les lieux paroissiaux.

Par ailleurs, tout nous porte à croire que, si nous en avions les ressources, une église ouverte tous les jours recevrait un flot de visiteurs. Beaucoup de gens nous en expriment le désir. L'été dernier, un étudiant a été engagé comme guide et animateur culturel. Aucune publicité n'avait été prévue. Mais il a suffi d'inscrire dehors sur une affiche "Église ouverte. Bienvenue ": en huit semaines, trois mille personnes ont visité les lieux. La plupart d'entre elles se sont accordé un temps de prière ou de méditation. Que venaient-elles chercher? Une émotion esthétique ou religieuse? Une oasis de paix et de silence? Une œuvre architecturale de grande beauté? Les nombreux commentaires reçus évoquent un peu tout cela. On y trouve exprimés des sentiments d'émerveillement, des échos d'une prière, des remerciements et des encouragements. Un jeune m'écrit : "Garde bien mon église en forme pour qu'un jour je vienne ici comme ton successeur». D'autres font part d'un projet de baptême ou de mariage auquel ce lieu offrirait une ambiance privilégiée. Plusieurs visites se prolongent en rencontres pastorales.

On comprend que l'ampleur de l'église, sa beauté et la qualité de son acoustique n'aient pas échappé au monde des musiciens. Dès mon arrivée comme pasteur, des facultés universitaires de musique, le Conservatoire du Québec, de nombreuses formations musicales m'exprimaient leur espoir d'une collaboration. Des producteurs, des chefs d'orchestre et des musiciens sont venus, désireux que l'on accueille leurs projets en les comprenant de l'intérieur. Tous ces liens n'ont fait que grandir depuis.

Il faut dire ici que l'église Saint-Jean-Baptiste est reconnue pour ses orgues: les grandes orgues, l'orgue de chœur et l'orgue de la chapelle. Il s'agit de trois instruments qui font, à juste titre, la fierté de la maison Casavant. Ils sont entretenus avec grand soin. Prochainement, par exemple, le grand orgue fera l'objet d'une restauration d'envergure. Les visiteurs en 
apprécient la qualité sonore mais aussi la beauté. Son buffet à lui seul suscite une attention émerveillée. La présence d'un organiste titulaire de la qualité de $\mathrm{M}$. Jacques Boucher a grandement facilité l'essor de la vie artistique à l'église. Une équipe responsable s'est formée autour de lui. Les concerts se sont multipliés. Certains ont connu des dimensions inespérées. Je pense entre autres à la Messe solennelle de sainte Cécile de Gounod que le Chœur et l'Orchestre Métropolitain ont interprétée pour l'émission "les Beaux Dimanches " à Radio-Canada, avec une télédiffusion dans vingt-cinq pays. Je n'oublie pas non plus des créations d'œuvres québécoises comme Avec (Wampum Symphonique) de Gilles Tremblay, radiodiffusée en grande première à travers le monde. Plus récemment, des chorales du Bas du fleuve et de la Gaspésie se regroupaient et s'adjoignaient l'Orchestre symphonique des jeunes de Montréal pour une "Grande virée ", un ensemble de concerts donnés à Paspébiac, puis à Saint-Pascal de Kamouraska, pour ensuite envahir l'église Saint-JeanBaptiste de Montréal dans un climat exceptionnel d'enthousiasme et de fraternité. Il faut souligner également la venue de grands ensembles de divers horizons : des bulgares, des tibétains, des chinois, des portugais, des russes, etc. Il nous a été particulièrement agréable d'accueillir les formations musicales de nos milieux universitaires. C'est ainsi que nous avons reçu l'Orchestre symphonique de l'Université de Montréal, l'Orchestre symphonique de McGill et le Chœur de l'UQAM.

Nous avons eu le bonheur de recevoir les membres de la Commission Radiophonique des Programmes de Langue Française, regroupant la Radio-Télévision belge francophone, Radio-Canada, Radio-France et la Radio suisse-romande. L'été prochain, la Rencontre internationale des chorales d'enfants (Pueri cantores) viendra vivre dans notre église un grand événement.

Des cinéastes et des gens de théâtre ont également manifesté leur intérêt pour l'église et le presbytère. À travers la présence d'un Jean Beaudin, d'un Robert Lepage, d'une Sylvie Payette ou d'un Pierre Nadeau en tournage chez nous, on développe des contacts avec un grand nombre de nos meilleurs artistes de la scène ou du cinéma.

Au plan religieux, on tient pour acquis que l'église Saint-Jean-Baptiste accueille au total chaque dimanche plusieurs centaines de personnes. Ce n'est pas un mince phénomène que le rassemblement d'une communauté fidèle, fervente et heureuse d'être au rendez-vous. Quelques fois par année, l'église se remplit à la faveur d'événements spéciaux : Noël, les Jours saints, la Saint-Jean, et un certain nombre d'assemblées de prière organisées par des institurions ou des groupes qui cherchent un grand 
espace et qui rassemblent aussi des milliers de personnes. $\grave{A}$ elle seule, la pastorale scolaire organise à l'église des activités spéciales auxquelles participent des centaines d'enfants et d'adolescents. Il arrive que des événements célébrés dans l'église se prolongent en fêtes populaires dans la rue. Les gens du quartier apprécient particulièrement l'exubérance des fêtes comme celle de la Saint-Jean-Baptiste.

Enfin, on n'en finirait plus de raconter la vie qui anime le presbytère. Au salon, on y retrouve fréquemment des jeunes qui préparent leur mariage ou le baptême de leur enfant. On rencontre des familles en deuil qui viennent partager leur peine. Un bon nombre de personnes, en particulier des jeunes, demandent un accompagnement spirituel personnel. Dans la mesure de notre disponibilité, nous cherchons à favoriser ces rencontres. À la salle à manger, la table nous paraissait beaucoup trop grande au début. Maintenant, avec parfois plusieurs convives souvent improvisés, nous sommes tout à fait à l'aise d'avoir un grand espace pour partager dans un climat franchement familial.

On a eu à se poser également la question d'un grand presbytère. Estce un phénomène dépassé et inadéquat? Faut-il envisager la transformation des lieux, une location, ou carrément une vente? Chez nous, quelques pièces sont disponibles pour des résidents ou des visiteurs. Depuis trois ans, nous avons partagé la vie de tous les jours avec un certain nombre de jeunes moines en stage d'études à Montréal. Les gens du milieu se sont attachés à eux, en particulier aux moines de Saint-Jean dont quelques-uns ont fait des séjours d'une année ou deux. Nos liens avec le monastère Saint-Benoît-du-Lac donnent aussi une couleur particulière à notre présence pastorale. Il en est de même de nos solidarités avec les cisterciens. Les moines se sentent chez eux ici. En partageant notre vie et nos préoccupations, ils nous aident à garder à cette maison une ambiance de recueillement, de vie intellectuelle et de fraternité. De plus, il nous arrive aussi de compter parmi les nôtres des hôtes de passage, prêtres ou laïcs.

L'autre jour, nous recevions une dizaine de détenus de Bordeaux qui venaient passer l'après-midi avec nous pour visiter l'église, entendre le Grand orgue, écouter notre chanteur en résidence, puis échanger avec nous durant deux heures autour de la table. Le soir du même jour, nous recevions une vingtaine d'étudiants de niveau collégial avec lesquels le dialogue fut des plus intéressants.

Faut-il dire que plus on reçoit, plus on est invité à aller sur le terrain des autres? Nous sommes conscients de cette responsabilité. L'apprivoisement doit aller jusqu'au bout. 


\section{Réactions de l'équipe pastorale}

On le voit, les demandes sont nombreuses et très diversifiées. Plusieurs réactions seraient possibles de la part des pasteurs, des responsables et des membres de la communauté chrétienne.

D'abord, le repli serait facile. Sous le prétexte de prudence et de respect des lieux, il suffirait de multiplier les refus. On évite à ce moment-là tout risque d'équivoque et toute critique. Cela comporte des avantages : d'abord la paix, puis le caractère nettement univoque de la signification des lieux. Par contre, les inconvénients méritent d'être considérés. Le danger nous guette de faire de nos temples des édifices coupés de la vie du quartier et peut-être même insignifiants, c'est-à-dire dépourvus de sens, pour la majorité des gens qui ne fréquentent pas notre communauté. Avec le temps, une telle absence d'engagement ne peut qu'accroître l'isolement de la paroisse et la confiner dans un statut marginal.

Une autre réaction serait relativement facile. Elle nous est d'ailleurs régulièrement proposée ou suggérée au nom du réalisme. Il s'agirait d'établir avec les personnes que nous accueillons des rapports de propriétaires à locataires. On accueillerait des groupes ou des individus d'abord en vue de percevoir des revenus qui aideraient à la bonne marche de la paroisse. De tels rapports ne suscitent chez ceux que nous accueillons aucun sentiment d'appartenance. Ils ne permettent aucune occasion de faire découvrir des liens possibles entre nous, ou encore des valeurs qui sont véhiculées dans ces lieux. Dans un tel contexte, il est assez indifférent de présenter à des milliers de personnes un Requiem de Fauré, une Messe de Gounod, ou plus simplement un bingo ou une activité lucrative quelconque. Nous avons renoncé à cette option. Les revenus générés par les concerts ne sont quand même pas négligeables. Ils représentent environ dix pour cent de l'ensemble de nos revenus. Il faut dire que l'on tient à s'assurer qu'un bon nombre de concerts soient gratuits et accessibles à tous les gens du milieu qui veulent y assister.

Nous avons rejeté également la tentation d'un rapport utilitaire avec les gens qui font appel à nous pour disposer de locaux. Pour nous, il n'est pas question de mettre en veilleuse l'esprit pastoral pour rendre nos locaux rentables et assurer à l'église une visibilité qui faciliterait des retombées économiques intéressantes. Tout comme il n'est pas question d'entretenir avec les gens qui nous fréquentent des rapports moralisateurs ou récupérateurs. 
Nous n'avons donc pas adopté une position de repli, comme si nous étions, dans la société actuelle, une forteresse assiégée. Nous n'agissons pas en propriétaires qui cherchent quels avantages ils pourraient tirer de leurs locataires. Avant tout, notre option très nette en est une d'accueil. Nous croyons fermement à cette attitude amicale et chaleureuse qui est celle même du Seigneur : "Venez et voyez " $(J n$ 1,9). Nous cherchons à être solidaires de leurs projets. Ces derniers demeurent les leurs, mais nous sommes heureux de nous y associer à cause de l'intérêt que nous y portons.

Cet accueil comporte des risques. Même si elle se montre très favorable à notre action, l'opinion publique a ses moments de nervosité. Certains réagiront devant l'un ou l'autre de nos engagements comme devant un scandale. Des personnes trouveront que nous pourrions faire de meilleures affaires si nous étions moins soucieux d'exercer un discernement pastoral. D'autres trouveront qu'on se donne beaucoup de mal et de peine quand ce n'est pas nécessaire. D'autres enfin pourraient avoir peur que le sens d'un lieu-église soit noyé dans un flot de significations quasi contradictoires. Ces préoccupations ne nous laissent pas indifférents. Nous partageons le souci que l'accueil se fasse dans la fidélité à la vocation du lieu.

À la faveur de l'expérience, une grande conviction nous habite. Lorsque des gens s'adressent à nous en vue de présenter telle ou telle activité, il y a là, surtout quand il s'agit de l'église, beaucoup plus qu'une simple demande de location. Plusieurs savent qu'ils retrouveront un cadre qui les touche et qui n'est pas sans dimension spirituelle ou transcendante. Par exemple, à l'occasion des grands concerts dans l'église, des centaines de contacts nous amènent à penser qu'il n'est pas indifférent pour quelqu'un de vivre quelques heures dans une belle église pour entendre une messe, un requiem ou un autre chef-d'œuvre qui élève le cœur et l'âme. On n'est pas impunément exposé à la beauté, à l'harmonie et à la paix d'un lieu spirituel sans être partiellement transformé.

La signification d'une église dans un quartier n'est pas qu'inscrite dans la pierre. Elle est exprimée aussi par le type d'accueil et de liens créés par les responsables du lieu avec toutes les personnes qui le fréquentent pour un motif valable. Quand il s'agit de musique, on ne demande pas aux pasteurs d'être des musiciens ou des mélomanes avertis. On attend d'eux des qualités d'accueil et de présence. Nous remarquons d'ailleurs que nous sommes portés à accueillir un chef d'orchestre comme nous le faisons pour un jeune itinérant. Il s'agit de la même ouverture d'esprit et de cœur, du même respect et de la même joie à devenir des amis. Même les gens les 
plus admirés et les plus applaudis ont besoin d'être reçus, compris, estimés, et accueillis joyeusement dans une ambiance chaleureuse. Les soirs de concerts, le presbytère est une grande maison ouverte. Les musiciens s'y sentent chez eux. Les pièces du presbytère deviennent des loges pour les vocalises des solistes et les gratifications d'après-concert auxquelles se mêle, pour certains artistes, beaucoup de solitude. La maison devient aussi un lieu d'échanges avec de nombreuses personnes chargées de hautes responsabilités qui côtoyent ici des gens du milieu dans un climat de fête.

Depuis quelques temps, plusieurs musiciens ont offert leurs services pour la liturgie du dimanche. Ils l'ont fait spontanément. Ils sont rattachés à la communauté chrétienne de Saint-Jean-Baptiste. En plus du Chœur grégorien, nous comptons une vingtaine d'étudiants en musique qui forment l'Ensemble vocal Saint-Louis, un quatuor vocal de jeunes musiciens de même qu'un jeune artiste en résidence, baryton de classe internationale. Ce dernier, venu comme soliste dans un grand concert Fauré, a créé des liens sympathiques avec les gens de la maison. Il a offert ses services pour la liturgie pour enfin manifester le désir de faire partie de la résidence. Les musiciens ne viennent pas aux célébrations pour donner des concerts. Ils se mettent au service de la liturgie et sont heureux de fréquenter le presbytère où ils trouvent chez les pasteurs des frères et des amis. De telles alliances ont rendu possibles des projets inédits comme, par exemple, la réalisation d'un disque-compact consacré à des réflexions sur l'Évangile. Ce sont peut-être là quelques fruits de l'hospitalité que nous cherchons à pratiquer.

Lorsque je me retrouve en silence dans notre église, je me dis souvent qu'il y a une grâce attachée à ce lieu. D'abord une grâce de beauté. L'écho de nos liturgies et de nos rassemblements me revient. Je me dis que si la justice, le partage et la vérité sont des chemins vers Dieu, la beauté aussi en est un qui peut nous mener à la rencontre du Dieu créateur de toutes ces choses qui provoquent notre émerveillement. Ce lieu sacré a une intentionnalité très nette : la rencontre de Dieu. Les concerts eux-mêmes s'en trouvent magnifiés! Une église qui accueille des artistes et s'ouvre pour devenir le lieu d'importants événements culturels, c'est tout autre chose qu'un temple qui renoncerait à sa vocation première pour devenir, à la suite d'une vente, une salle comme une autre. Il n'y aurait plus d'évocation de la foi, du sacré ou de la transcendance. Il n'y aurait plus ce renvoi à un "ailleurs". Entrer dans cet ailleurs, ce n'est pas gérer le passé, mais prendre le risque d'une rencontre vivante dans un lieu qui, pour des centaines de personnes, constitue bien celui de la Rencontre. Cet ailleurs n'est pas seulement un passé, mais un présent, bien plus un Autrement et aussi un Autre. 
Après de telles considérations, on comprend qu'on ne peut faire dans une église n'importe quoi. Les choix se heurtent parfois à des frontières difficiles à définir. Mais le répertoire des concerts, par exemple, tiendra compre du lieu. Ainsi faisait récemment le Requiem de Verdi en mémoire des jeunes femmes victimes de la tuerie à l'École polytechnique en 1989. Il arrivera cependant que des groupes soient reçus, qui ne font pas l'unanimité. Je me rappelle l'accueil qu'après des consultations sérieuses nous avions fait à des moines tibétains venus révéler en spectacle leur tradition spirituelle. Les échanges avaient été exceptionnels, surtout à l'heure où nous partagions le thé, comme on se livre à un rituel. Tous les bouddhistes de la région de Montréal s'étaient intéressés à cet événement et avaient été touchés par l'ouverture du milieu. Ce fut un grand moment de dialogue inter-religieux.

Chaque groupe qui est reçu à Saint-Jean-Baptiste est d'abord accueilli dans un dialogue qui nous permet de partager ses centres d'intérêts, ses problèmes et ses projets. À la longue, une telle attitude a force de référence pour des personnes et des organismes qui cherchent des lieux de dialogue dans l'Église. L'intérêt des médias en particulier est manifeste. Un discernement s'impose, mais aussi une certaine audace évangélique.

Au début de mon ministère comme jeune prêtre, en 1960, la signification des édifices religieux était univoque. Toutes les activités qui s'y déroulaient relevaient de nous. Et elles avaient un lien évident avec l'évangélisation et la pastorale des sacrements. Aujourd'hui, les mêmes lieux acquièrent une signification qui suppose des attitudes d'accueil, de dialogue et de partenariat. Non seulement les lieux connaissent des usages plus nombreux et diversifiés, mais leur impact symbolique dans le milieu prend un relief nouveau. Quand il s'agit de concerts à l'église par exemple, il est clair qu'on se trouve dans un lieu habité, un lieu sacré que fréquente assidûment une communauté de croyants et de croyantes. Ce sont eux qui ouvrent leurs portes et qui accueillent d'autres croyants ou encore des gens qui ne partagent pas nécessairement leurs convictions, avec les talents qui sont les leurs. Même quand il s'agit de grands événements artistiques, les ancrages traditionnels, les points de repère demeurent. Les croyants sont heureux de s'y retrouver. Les autres se laissent toucher par cet accueil et par la beauté des lieux.

Au cours de l'année, plusieurs dizaines de milliers de personnes fréquentent notre église et ses locaux. Les saisons musicales attirent environ cinquante mille personnes qui viennent vivre ici des heures privilégiées dans un cadre qu'elles admirent. Nous n'avons pas cherché à provoquer cet état de fait. D'une certaine manière, il s'est imposé à nous. Devant toutes 
les demandes reçues et les désirs manifestés, nous nous sommes demandé quoi faire. Grâce à de nombreuses collaborations avec des gens capables de réfléchir à ce phénomène et de trouver des moyens concrets de réalisation, nous avons élaboré une programmation intéressante, accordée à la dignité du lieu.

Des paroisses environnantes réagissent de manière analogue. Je pense ici à Saint-Denis, dont le pasteur est doué d'une créativité hors du commun et qui a su faire équipe avec un grand organiste, M. Réjean Poirier. Avec une équipe de bénévoles, ils arrivent à des réalisations de qualité. De plus, ce curé, Pierre Desroches, avec sa riche expérience auprès des personnes démunies et handicapées, réussit à multiplier les initiatives sociales. Il abrite en particulier ce qu'on appelle le Resto-Plateau, un service communautaire qui offre le repas à des centaines de personnes chaque jour. Dans une autre paroisse, Saint-Louis-de-France, le sous-sol est mis à la disposition de l'Abri des jeunes, un centre pour jeunes itinérants. Il s'y fait des merveilles grâce à la compétence des personnes responsables. Le curé, Jean-Maurice Thérien, nous interpelle toujours lorsqu'il nous parle des dynamismes de son milieu. Au centre-ville, quelques paroisses de confessions religieuses diverses nous inspirent particulièrement. Nous avons avec leurs responsables des échanges précieux. Je citerai simplement quelques lieux qui nous sont familiers: Christ Church Cathedral, StJames United Church, St-James The Apostle.

Chaque édifice religieux, avec ses ressources diverses, représente un outil important pour qui peut s'en servir. Il m'arrive de dire que nous disposons d'un stradivarius en pastorale. On peut être embarrasé par un instrument aussi précieux et d'aussi grande qualité. On peut, au contraire, y trouver un défi qui nous met, bien sûr, à l'épreuve, mais qui permet d'accomplir de grandes choses. Au cœur de la ville,de tels Stradivarius constituent une richesse non seulement ecclésiale et pastorale, mais également une richesse urbaine: des pierres vivantes et vibrantes au cœur du béton du centre-ville.

\section{Pistes de réflexions}

Autrefois, l'église étair le lieu de liturgies, de sacrements et d'activités spirituelles bien définis, que l'on retrouvait d'une paroisse à l'autre. Aujourd'hui encore, ces nécessités demeurent, incontestablement. Il nous faut toujours une communauté au sein de laquelle se vivent les célébrations de baptêmes et de mariages, les funérailles chrétiennes, les liturgies dominicales, les grands rassemblements de prière, etc. Beaucoup de personnes viennent de façon ponctuelle. Mais, bien des situations 
inédites nous obligent à créer de nouvelles façons de répondre aux besoins pastoraux. Dans ce contexte nouveau, nous devons nous assurer que le lieu sacré sert aussi de prélude à l'évangélisation, de préparation à l'accueil de la bonne nouvelle, quels que soient les événements qu'on y vit.

Comment assurer cette préparation évangélique? Nous estimons que l'homme et la femme des grandes villes demeurent désireux de paix, de silence, de prière et d'intériorité. Les croyants sont beaucoup plus nombreux qu'on pense qui veulent se retrouver dans une église pour s'adonner à la prière. Et plusieurs de ces croyants, à notre avis, sont de grands contemplatifs. Il y a ici des saints et des saintes authentiques, nous en sommes sûrs. Cela aussi fait partie du paysage urbain.

Il est bon alors de se rappeler qu'un temple comme Saint-JeanBaptiste représente un grand édifice patrimonial qui évoque la permanence dans un monde en mouvance constante. Les gens sont préoccupés de sens. Ils ne viennent pas à l'église seulement à cause de préoccupations d'ordre architectural, mais bien pour retrouver leurs racines. Beaucoup de jeunes demandent de venir prier, dessiner ou encore prendre des photographies. Ils viennent ensuite causer de ce qu'ils ont pu admirer. Ils cherchent des clés de compréhension pour un agir dans lequel ils s'engageront parce qu'ils y auront trouvé du sens. Enfin, on constate une redécouverte et un attachement de plus en plus profond à notre patrimoine, en particulier notre patrimoine religieux qui compte parmi les plus beaux trésors du Québec. Beaucoup d'anciens et d'anciennes de la paroisse aiment venir nous rendre visite et évoquer les grands moments de leur vie qu'ils ont célébrés dans cette église. L'autre jour, un des jeunes détenus, venu avec ses compagnons, s'est recueilli sur la fontaine de son baptême et tout le groupe s'est rassemblé autour de lui. On sentait un geste grave rendu possible à cause d'un trésor qu'on avait eu soin de conserver.

On se surprend de voir des jeunes poser beaucoup de questions au sujet de l'orfèvrerie ancienne et des vêtements sacrés d'autrefois. Ils veulent voir. Ils veulent toucher. C'est toujours l'occasion d'un dialogue et souvent d'une catéchèse à saveur liturgique.

Dans la poétique urbaine, y aura-t-il place pour des lieux de paix, de silence et d'ouverture à la transcendance et à la foi? Quand l'anonymat enferme les gens dans un isolement douloureux, trouveront-ils des communautés marquées par des liens interpersonnels sympathiques et chaleureux? Avec toutes les misères auxquelles on se heurte dans une grande ville, trouveront-ils des groupes ou des personnes capables de créer des contacts, d'apporter une aide, de leur faire découvrir qu'ils sont importants aux yeux des autres, qu'ils font partie d'une grande famille? Dans l'agita- 
tion de nos milieux en quête de valeurs, nos églises sauront-elles faire découvrir un Ailleurs, et pourquoi pas l'Autre qui l'habite? Qu'il s'agisse de patrimoine, de culture, de sentiments d'appartenance, de sens de la vie ou de la foi, un grand temple au cœur de la ville représente une référence essentielle. 